\title{
Hoja de cálculo como estrategia didáctica para la compresión de la cinética química
}

\author{
Spreadsheet as a teaching strategy for the compression \\ of chemical kinetics
}

\author{
Génesis Idelmary Posada Gutiérrez \\ idelmary3@gmail.com \\ Código ORCID: 0000-0003-3312-001X
}

\author{
Obelis Yulibeth Semprún Chourio \\ obelisyuli@gmail.com \\ Código ORCID: 0000-0002-8987-9474
}

Ministerio del Poder Popular para la Educación, Venezuela

Recibido febrero 2020 / Arbitrado marzo 2020 / Publicado mayo 2020

\section{Resumen}

La presente investigación tuvo como objetivo general proponer estrategias didácticas a través del diseño de hojas de cálculo dirigidas a la comprensión de la cinética química a nivel de cuarto año de la U.E. "Hipólito Cisneros". Sustentado en las teorías de aprendizaje de Ausubel y Piaget y enmarcado bajo la modalidad de proyecto factible. Se aplicó un cuestionario dicotómico de 17 preguntas, fue sometido a la validez entre expertos y se midió la confiabilidad a través de la fórmula de Kuder-Richardson, el instrumento fue aplicado a una muestra de 12 estudiantes. Se procedió a analizar los resultados, estudiar la necesidad de implementar esta herramienta para la comprensión de la química, así como el estudio de la factibilidad del mismo, tomando en cuenta que la mayoría de los encuestados están de acuerdo con la implementación de esta estrategia didáctica para la comprensión de la cinética química.

\footnotetext{
Abstract

This research had the general objective of proposing teaching strategies through the design of spreadsheets aimed at understanding chemical kinetics at the fourth year of the U.E. "Hipólito Cisneros". Based on the learning theories of Ausubel and Piaget and framed under the feasible project modality. A dichotomous questionnaire of 17 questions was applied, it was sometimes valid among experts and reliability was measured through the Kuder-Richardson formula, the instrument was applied to a sample of 12 students, the results were analyzed, the need to implement this tool for the understanding of chemistry, as well as the study of its feasibility, taking into account that the majority of the respondents agree with the implementation of this didactic strategy for the understanding of chemical kinetics.
}

Palabras clave:

Hoja de cálculo; TIC; aprendizaje; cinética química

\section{Keywords:}

Spreadsheet;

TIC; learning; chemical kinetics 


\section{INTRODUCCIÓN}

$\mathrm{E}$

l surgimiento de la tecnología data en tiempos remotos al momento en el cual se convertían los recursos naturales en herramientas simples que facilitaban el trabajo en diversas ocupaciones, pero el concepto tecnología surgió a mediados del siglo XVIII entre los años 1751 a 1772, luego de nacer las primeras corrientes tecnológicas en los países Francia y Alemania. La tecnología se ha convertido en una fuente primordial en la economía y en el desarrollo de las sociedades del siglo XXI. De este mismo modo, con las Tecnologías de la Información y Comunicación (TIC), comenzaron con la llamada sociedad de la información.

En los últimos 20 años la incorporación de las TIC en el aula se ha basado simplemente en la introducción de dispositivos y no se tiene claridad de los fines y estrategias pedagógicas para darle un funcionamiento útil, solo ocupan un lugar en las instituciones educativas, lo que implica un desafío pedagógico el cual muchos docentes no están preparados para afrontar, aunque la integración de las mismas es aceptada en los procesos de enseñanza y aprendizaje no muchos están satisfechos con el uso de ellas.

En América Latina los países han realizado muchos cambios a nivel educativo para la introducción de las Tecnologías de información y comunicación, en el año 2010 gobiernos de países como Chile, Costa Rica, India (Kerala) el cual es uno de los mayores programas que utiliza software de código libre, se dedicaron a la utilización de las mismas en el ámbito educativo. Asimismo, Israel es un país que en los últimos años ha buscado ser una potencia mundial debido a sus numerosas inversiones en ciencia y tecnología, favoreciendo su desarrollo, es el segundo país con más potencial tecnológico a nivel mundial, el primero es Estados Unidos, el éxito que ha tenido se debe a que tienen un excelente sistema educativo adoptando el conocimiento innovador y tecnológico como pilar fundamental para su formación.

En la actualidad y debido a la revolución de las nuevas tecnologías, la sociedad ha atravesado por grandes cambios significativos como la masificación de los medios de comunicación, el uso de la Internet como medio para la transferencia de información de manera inmediata entre una red de personas, lo cual parece traer atractivas soluciones principalmente para la educación, lo que implica que en este ámbito se implemente nuevas herramientas para la 
enseñanza y aprendizaje, la tecnología ha avanzado en un gran nivel, desde la década de los 70 surge los aparatos electrónicos (computadores, teléfonos, televisores, entre muchos otros) son incontables y con usos muy variados, pero es en los años 80 cuando surgen las telecomunicaciones, es decir, la convergencia entre la electrónica y la informática para establecer intercomunicaciones entre los individuos.

La tecnología a su vez ha tenido un impacto en la sociedad en los últimos años y los docentes deben introducir el uso de las mismas en el proceso de enseñanza, se debe innovar e ir a la vanguardia de la era digital en la que se está viviendo, así como, comprender las necesidades de la sociedad del siglo XXI, en este sentido la Organización para las Naciones Unidas para la Educación, Ciencia y la Cultura en sus siglas Unesco (2013) plantea: "Los sistemas escolares se ven enfrentados así a la necesidad de una transformación mayor e ineludible de evolucionar desde una educación que servía a una sociedad industrial, a otra que prepare para desenvolverse en la sociedad del conocimiento" (p.15).

En Venezuela el analfabetismo tecnológico se ha convertido en el principal factor por el cual los docentes se limitan a implementar el uso de las herramientas como las TIC en la enseñanza de las ciencias. Es por ello que se busca promover el uso de los Entornos Virtuales de Enseñanza y Aprendizaje (EVEA), tanto en los docentes que enseñan como en los estudiantes. Por lo tanto, el empleo de estos espacios para la enseñanza de las ciencias, especialmente en la química, constituye una herramienta fundamental para que el estudiante se relacione de manera más amigable con los contenidos impartidos, escenario en el cual, se conduce a este al conocimiento para un mejor desenvolvimiento en el área. La aplicación de este mecanismo (EVEA) a través de la tecnología mejora y favorece el desempeño del estudiante buscando como objetivo principal el interés de manera personal. Con relación a lo expuesto anteriormente, se presume que el empleo de esta herramienta didáctica serviría de gran ayuda para obtener un mejor resultado del aprendizaje de las ciencias, facilitando de manera eficaz la resolución de los problemas que se presenten en el menor tiempo posible. De hecho, la tecnología en la enseñanza cuenta con una amplia gama de aplicaciones, los cuales en su época eran novedosos. En la actualidad la tecnología avanza en una gran escala, y se ha convertido en una parte fundamental del día a día de los seres humanos. 
Por lo tanto, los docentes deben integrar esta valiosa herramienta en las aulas de clase, para facilitar la comprensión de los contenidos y motivando a los estudiantes para que el aprendizaje sea significativo, así mismo, una de las principales fortalezas de implementar el uso de la tecnología en la enseñanza es, que mejora las destrezas y habilidades permitiendo el aprendizaje colaborativo y participativos entre los alumnos, aumentando su capacidad de razonamiento y proporcionando una formación de calidad.

Las simulaciones con las hojas de cálculo son una herramienta que tienen grandes ventajas, y una de ellas es que no necesita de una comprensión amplia del lenguaje de programación, sino que resulta fácil utilizarlas, Raviolo. (2011) menciona: "una de las principales ventajas de operar con una hoja de cálculo es que el usuario puede realizar tareas de programación sin necesidad de dominar un lenguaje específico. Además que su funcionamiento básico es estable en el tiempo" (p.357); es decir, a pesar de sus actualizaciones sus funciones básicas se mantienen, son un recurso que se encuentra instalado en cualquier computador y que ayuda al desarrollo de habilidades y destreza en el estudiante.

Para Carso (citado en Andrés Raviolo, 2011) "la utilidad de una hoja de cálculo para la enseñanza de las ciencias está más limitada por nuestra imaginación que por el potencial en software". (p.506), por ende, no se requiere de un conocimiento abstracto de la informática, ni del uso de una hoja de cálculo, sino de integrar las herramientas tecnológicas en la enseñanza de la química. Por lo tanto, el uso de las TIC en las aulas de clase sirve como herramienta para hacer el contenido comprensible, desarrollando en los estudiantes habilidades para la interpretación de datos, resolución de problemas y la búsqueda de estrategias que le ayuden el proceso de aprendizaje.

En atención a las observaciones realizadas en la institución objeto de este estudio, se pudo denotar la falta de aplicación de estrategias didácticas fundamentadas en la tecnología de información y comunicación; por lo que se sigue trabajando de manera tradicional solo con el uso de la pizarra. De hecho, los avances tecnológicos son cada vez más vertiginosos; siendo la tarea del docente orientar a los estudiantes en el manejo de las mismas.

Es por ello que la utilización de la hoja de cálculo para la comprensión de la química fomentaría en gran manera el aprendizaje significativo, ya que las clases en el área de la química se abordan de forma teórica, sin dar espacio para la práctica; en este sentido, con la 
aplicación de esta estrategia didáctica, se pretende que los estudiantes comprendan de manera amigable el contenido de la cinética química.

Del tal forma, la utilización de esta estrategia didáctica en los estudiantes generaría curiosidad; siendo esta de manera provechosa para el docente en aula, obteniendo mejores resultados en su ámbito y rendimiento educativo, afianzando cada día más de manera práctica y que se interesen por aprender, indagando por si solos, obteniendo interrogantes y respuestas a las mismas, la relación docenteestudiante va surgiendo de manera fluida en donde el docente observaría resultados satisfactorios generados a través de la utilización de esta herramienta en el aula. Cabe destacar que la U.E "Hipólito Cisneros" cuenta con un salón de computación, el cual tiene a disposición 14 computadoras en perfecto funcionamiento y que están abiertas al acceso de los estudiantes de esta institución, de este modo esta unidad educativa cuenta con los medios necesarios para poner en práctica la propuesta para el diseño de una hoja de cálculo para la comprensión de la química.

Finalmente, con la utilización de la hoja de cálculo sería una gran opción para el docente en los diferentes temas abordados en química, podrían ir incluyéndola como estrategia dinámica para una mejor compresión en el área en donde se observe que al estudiante se le dificultad más la aprehensión del contenido, así mismo, es importante plantear la siguiente pregunta de investigación ¿Qué tan necesario será la implementación de una hoja de cálculo como estrategia didáctica para la enseñanza de la química?

Para dar respuesta a esta interrogante se planteó como objetivo general de la investigación proponer estrategias didácticas a través del diseño de hojas de cálculo dirigidas a la comprensión de la cinética química a nivel de cuarto año de la U.E. "Hipólito Cisneros". Para el logro del mismo, se hizo necesario en primer término, diagnosticar la necesidad del diseño de una hoja de cálculo como estrategia didáctica en la enseñanza y aprendizaje de la cinética química, seguidamente determinar la factibilidad de la propuesta y así diseñar una hoja de cálculo como estrategia didáctica en la enseñanza y aprendizaje de la cinética química.

Vale decir entonces, esta investigación se justificó debido a que el estudio de las ciencias es fundamental en todo ámbito, ya sea educativo, social, económico, pues las investigaciones científicas van avanzando, junto con el desarrollo de un país; por este motivo, los 
países emergentes o conocidos también como países en vía de desarrollo deben su lento proceso a que el estudio de las ciencias es poco, existe en Venezuela diferentes razones por la cual a los estudiantes les resulta tedioso incursar en el mundo de la ciencia, principalmente porque no hay motivación, se ve como un proceso muy abstracto y de difícil comprensión, a cambio de eso se prefiere escoger una carrera rápida sin hacer un mayor esfuerzo. Países como, Israel, Alemania, Estados Unidos, deben su desarrollo a que sus inversiones en ciencia y tecnología han sido numerosas y las cuales se han realizado, principalmente, a través de la educación.

Por lo tanto, la implementación de las TIC en la educación, en especial en la Educación Media General, buscó la manera que el estudiante conozca nuevas estrategias de aprendizaje, las cuales promuevan el interés por el estudio de las ciencias, cabe destacar que, en el área de la química se realizan muchos procesos químicos los cuales necesitan la aplicación de cálculos y análisis de los resultados que a los discentes se les dificultad comprender; por lo tanto, se tiene como intención primordial brindar una herramienta que fomente la motivación y el incentivo a los estudiantes para que se interesen cada día más por el estudio de las ciencias con ayuda de las tecnologías de la información. La UNESCO (ob. cit.) por ejemplo señala:

La alfabetización digital describe las habilidades básicas relativas a las TIC que toda persona debe manejar para no ser/estar totalmente excluido...La escuela como espacio formal de educación con asignaturas, aulas y espacios/tiempo de enseñanza aprendizaje. requieren ser transformados para ser más permeables y dinámicos. (p. 17)

Desarrollando así nuevas prácticas educativas que generen el interés y la motivación de cada uno de los aprendices, la tecnología en el siglo XXI no se puede desligar de las sociedades y es por ello que desde la educación se debe fomentar el uso de las herramientas tecnológicas que se encuentran al alcance de las personas. Por consiguiente, esta investigación se enmarcó bajo el área prioritaria "Educación, didáctica y gerencia aplicada" dentro de la línea de investigación "Pedagogía, andragogía y gerencia aplicada a la Biología y la Química", adscrita al departamento de Biología y Química de la Faculta de Ciencias de la Educación. 


\section{MÉTODO}

$\mathrm{E}$ n cuanto a la ruta metodológica se detallan cada uno de los aspectos relacionados con el desarrollo de la investigación, se trata de los pasos que el investigador debe llevar a cabo para impulsar su estudio como lo son: diseño de la investigación, tipo, nivel, técnica de recolección de datos, población, muestra, la validez y confiabilidad de la misma, todos estos aspectos permiten reconocer la realidad de los sujetos a explorar, son los métodos y técnicas utilizados por el investigador para llegar cumplir con los objetivos propuestos. Hurtado y Toro (2007) afirman "se refiere al desarrollo propiamente del trabajo investigativo" (p.90).

Mientras la naturaleza de la investigación se enfocó del enfoque de manera cuantitativa tal como lo definen Hernández, Fernández y Baptista (2014): "utiliza la recolección de datos para probar hipótesis con base a la medición numérica y el análisis estadístico. Con el fin de establecer pautas de comportamiento y probar teorías" (p.4). En este enfoque las variables son susceptibles a medición, sus resultados son numéricos y probatorios a través del uso de la estadística, analizando y describiendo la realidad de forma objetiva y secuencial. En este sentido, la naturaleza de la realidad no cambia con la visión del investigador para ello los sentimientos y creencias deben dejarse a un lado y ser totalmente objetivas para no influir en el resultado de los análisis de la recolección de datos.

Por ende, el presente estudio se centró en el diseño no experimental de campo, con un alcance descriptivo, ya que, se describen, fenómenos, sucesos, tal y como se manifiestan. Arias (2006), define el nivel de investigación como aquel que; "Se refiere al grado de profundidad con los que se aborda un objeto o un fenómeno, aquí se indicara si se trata de una investigación exploratoria, descriptiva o explicativa" (p.54). Este depende de los objetivos que pueda tener la investigación, pues se describió los conocimientos y nociones que poseen los estudiantes en cuanto a las tecnologías de informaciones y comunicación así como su empleo en el estudio de la Química. Durante mucho tiempo se han realizado propuestas para el uso de las TIC como complemento de las actividades en el aula de clase, de esta manera se da continuidad al proyecto factible sobre el diseño de una hoja de cálculo para la comprensión de la cinética química. 
La modalidad de investigación trata de aquellos modelos que se utilizan para llevar cabo un estudio. El presente se enmarcó bajo la modalidad de proyecto factible, dirigida a la elaboración de una propuesta de modelo operativo para el desarrollo de los requerimientos y necesidades observadas. Arias (ob. cit.) define: "es una propuesta de acción para resolver un problema práctico o satisfacer una necesidad. Es indispensable que dicha propuesta se acompañe de una investigación que demuestra factibilidad o posibilidad de realización" (p.134). Se le llama factible porque atiende a necesidades específicas de un grupo de personas y son posibles de realizar donde los gastos económicos son mínimos.

En cuanto a la población, ésta se conformó por 33 estudiantes pertenecientes al cuarto año de educación media general, de acuerdo a los definido por Rojas (2010) "una población está conformada por un grupo de personas que cumplen con ciertas características de interés para una investigación" (p.126); es decir que concuerdan con las mismas especificaciones, situándose en la misma características de contenido, lugar y tiempo, la población es la unidad que se dese estudiar, por lo general es un número inaccesible, por lo que se elige un muestra que represente a la población total. Mientras la muestra fue de 12 estudiantes de cuarto año de esta institución, tal como lo define Palella y Martins (2012): “Un subgrupo de la población de interés sobre la cual se recolectan datos, y que tiene que definirse y delimitarse de antemano con precisión, además de ser representativo de la población" (p.173).

Por ende, la muestra es una la toma de una parte de la población a la cual se estudiara, esta tiene que ser representativa. Las muestran se clasifican en dos dimensiones; las probabilísticas (todos tienen la misma posibilidad de ser elegidos) y las no probabilísticas (depende de la característica de investigación). Como técnica para la recolección de datos fue considerada la encuesta; la cual en palabras de Guffante et al. (2016) en sí son cuestionarios que:

se utilizan para obtener información de una muestra, existen dos tipos; la primera es la oral, la cual, se trata de un interrogatorio y se vale de recursos como grabadoras o videocámaras, es más profunda y segunda es la escrita que a diferencia de la oral se entrega un cuestionario a la persona el cual debe responder de acuerdo con las indicaciones del encuestador. Suelen ser del tipo cerradas, abiertas y mixtas. (p.97) 
Dicha encuesta estuvo conformada por opciones de respuestas cerradas de carácter dicotómico. De hecho, Hernández et al. (2014), manifiestan; "Los instrumentos para la recolección de datos es un recurso que utiliza el investigador para registrar información o datos sobre las variables que tiene en mente" (p.199), los cuales pueden ser, cuestionarios, guías de entrevista, lista de cotejo, escala de estimación, entre otros.

De manera pues los cuestionarios consisten en un conjunto de preguntas para obtener información necesaria, obteniendo como objetivo preguntas concretas que serán respondidas por las personas encuestadas. Dicho instrumento se constituyó por 17 preguntas. La validez del mismo se realizó a través del juicio de expertos bajo una revisión del contenido, criterios y construcción del mismo para verificar si ellos se justaron al estudio, el mismo fue entregado a tres profesores de la Facultad de Ciencias de la Educación de la Universidad de Carabobo, quienes se encargaron de revisar la redacción de los ítems, el vocabulario empleado, el dominio de contenido y si el instrumento es congruente y mide realmente los lo que se desea medir. Del mismo modo, los expertos hicieron sus observaciones y se corrigió lo necesario y de esta manera dieron su aprobación para ser aplicado.

Para la confiabilidad que no es más que el grado con el sometido a varias repeticiones este pueda dar un resultado igual en el mismo individuo, si los resultados no son paralelos el instrumento no se puede tomar como confiable, ya que existe dispersión en los resultados. El nivel de confiabilidad deber estar en un rango mayor o igual a 0,62 . Para medir la confiabilidad del instrumento se hizo uso del modelo del coeficiente de Kuder-Richardson. Para el presente estudio se realizó una prueba piloto con 10 estudiantes, el rango de confiabilidad fue de 0,62 entra en el rango entre 0.61-0.80, el cual según Palella y Martins (ob. cit.) es de dimensión alta y que por lo tanto el instrumento es aplicable, ha sido medido con la fórmula de Kuder-Richardson utilizando una base de datos con la ayuda del programa Excel 2013.

Finalmente, la técnica de análisis de datos representa la forma de cómo será procesada la información, esta comprende clasificación, codificación a fin de llegar a conclusiones específicas. De acuerdo con 
los objetivos planteados y el procedimiento de la investigación se hace uso de la estadística inferencial, ya que, solo se recaban datos de una parte de la población, es decir, de una muestra y de allí hacer generalizaciones acerca de la totalidad de la población.

\section{RESULTADOS Y DISCUSIÓN}

A continuación se muestran los resultados obtenidos en el 1 diagnóstico realizado a los estudiantes del cuarto año donde se 1 detectó la necesidad del diseño de una hoja de cálculo como estrategia didáctica en la enseñanza y aprendizaje de la cinética química. Luego de terminar la fase de recolección de datos, se procedió a tabular los resultados con la ayuda del programa Excel 2013, utilizando la tabla de frecuencia y gráficos que permitan traducir la información cuantitativa proporcionada en interpretaciones, ya que la misma representa respuestas emitidas por los encuestados de acuerdo a la dimensión y tipo de indicadores. Por ende los resultados representados en los gráficos expresan los ítems agrupados de acuerdo a las dimensiones establecidas. En cuanto a la dimensión Conocimiento, se tienen agrupados cuatro ítems en los cuales se midió todo ese saber en torno a TIC, hoja de cálculo, cinética química, velocidad de reacción que tenían los estudiantes; en el gráfico 1, muestra los resultados.

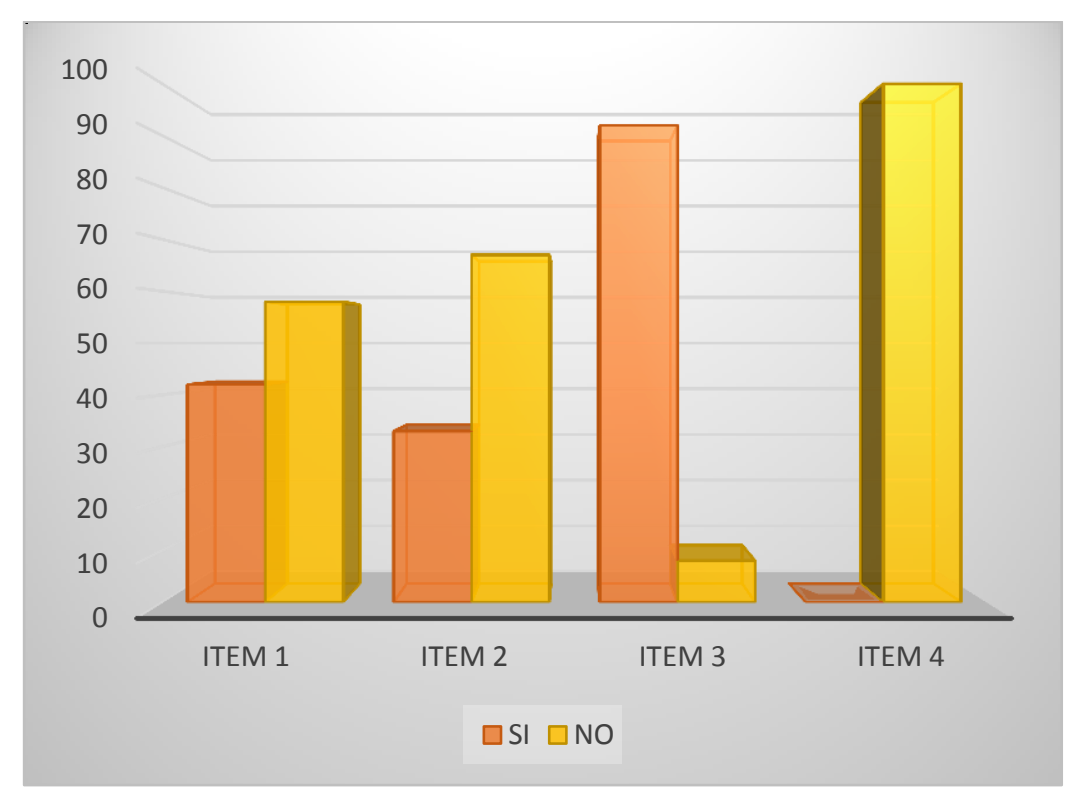

Gráfico 1. Relación porcentual entre los ítem 1, 2, 3, 4 (Fuente: Posada y Semprún, 2020) 
En los resultados obtenidos que se observa en el gráfico 1 que en el ítem 1 al menos 5 personas las cuales representan un 42 por ciento de la muestra contestaron conocer la cinética química como área de la fisicoquímica, mientras que un 58 por ciento respondió negativamente; del mismo modo, en el ítem 2 un 33 por ciento contestó que sí saben cómo calcular la velocidad de un reacción química, mientras que un 67 por ciento contestó que no conocer. En el ítem 3 un 8 por ciento afirmó haber visto el contenido de cinética química en la asignatura y un 92 por ciento respondió que no. Asimismo, en el ítem 4 el 100 por ciento de los estudiantes encuestados indican que no conocen lo que significa TIC.

De acuerdo con los estos resultados, arrojan que la mayoría de los encuestados desconocen que es la cinética química y como calcular la velocidad de reacción y no vieron el tema durante la asignatura, aunque en la trasformación curricular, el tema de cinética química, constituya uno de los ejes integradores de la asignatura, los profesores prefieren integrarlo con otros contenidos afín. Del mismo modo, todos los estudiantes desconocen lo que significa TIC, siendo esta la herramienta de comunicación más usadas por la población mundial.

En cuanto a la dimensión viabilidad, se buscó medir el acceso que tienen los estudiantes a la herramienta informática; por lo que en el Gráfico 2, presentado a continuación se expresan los resultados obtenidos.

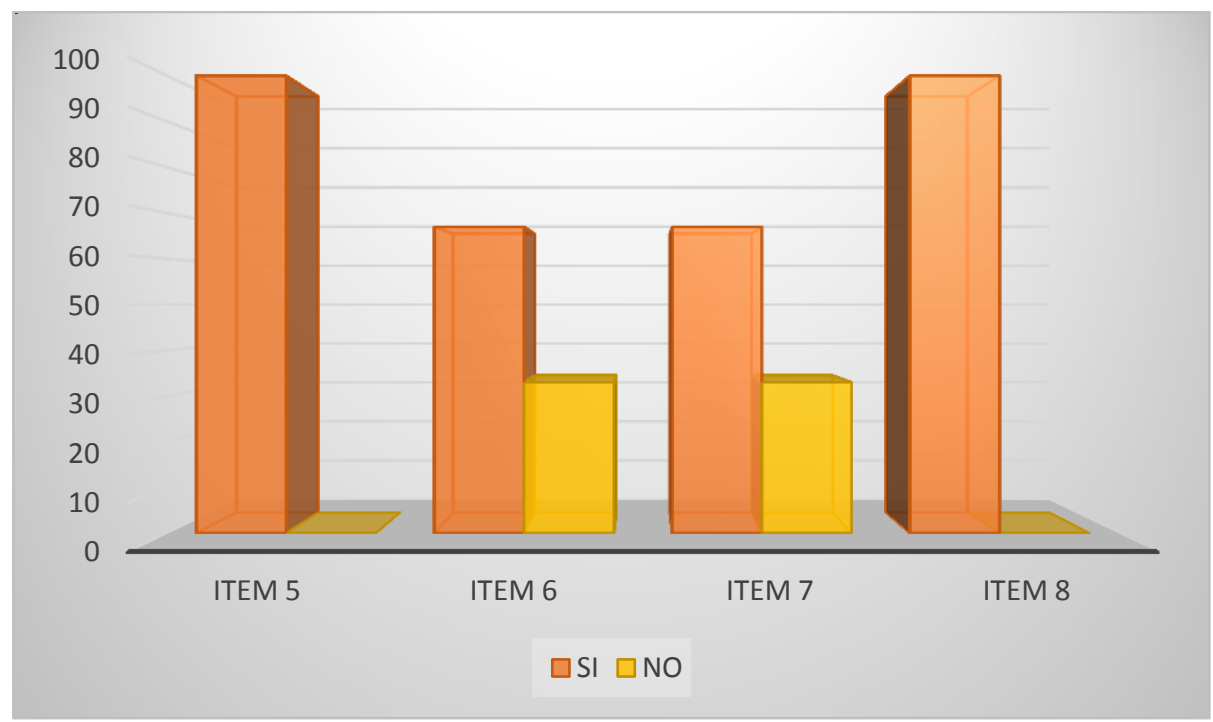

Gráfico 2. Relación porcentual entre los ítem 5, 6, 7, 8. (Fuente: Posada y Semprún, 2020) 
En los resultados representados en el gráfico anterior, se puede decir en cuanto al ítem 5, 100 por ciento de los estudiantes confirmaron que sí tienen computadora en casa; en el ítem 6 un 67 por ciento respondieron contar con acceso a internet pero hay un 33 por ciento que no; del mismo modo, en el ítem 7 un 67 por ciento de la muestra declaró que le dedica más de 3 horas al uso de la computadora en casa, mientras un 33 por ciento no. En el ítem 8 el 100 por ciento confirmó que dentro de la institución existe un laboratorio de computación. Por otra parte en la dimensión Utilidad, se buscó determinar el aprovechamiento del beneficio de la herramienta por parte de los estudiantes; los resultados se pueden apreciar en el Gráfico 3.

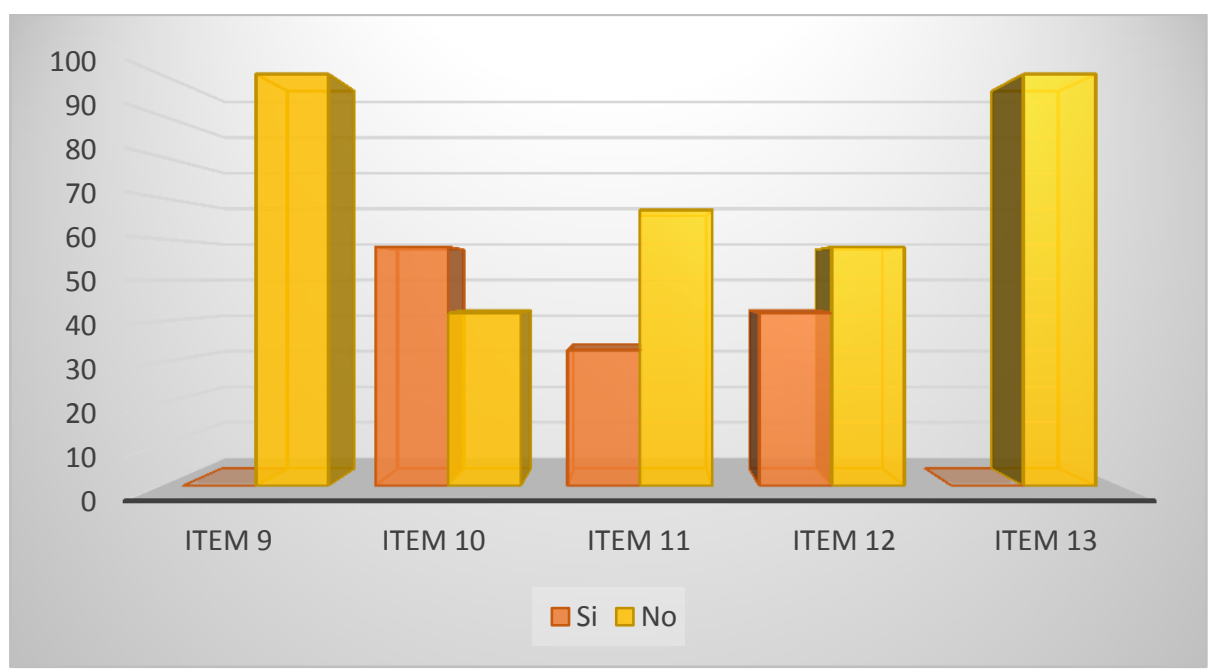

Gráfico 3. Relación porcentual entre los ítem 9, 10, 11, 12, 13. (Fuente: Posada y Semprún, 2020)

En el gráfico anterior, se puede evidenciar que en el ítem 9 se indica que un 100 por ciento de la población niegó utilizar el laboratorio de computación con frecuencia. Mientras, en el ítem 10 un 58 por ciento afirmó haber utilizado alguna vez una hoja de cálculo, pero un 42 por ciento reveló lo contrario.

En el ítem 11 un 33 por ciento sí manejan el uso de una hoja de cálculo, pero un 67 por ciento no. Asimismo en el ítem 2 un 42 por ciento ha trabajado con herramientas tecnológicas dentro de la institución, pero un 58 por ciento no. En el ítem 3 un 100 por ciento de la muestra niegó que actualmente en la institución utilicen las TIC en alguna asignatura. 
Por consiguiente, en la dimensión viabilidad, la totalidad de los estudiantes cuenta con computadora en casa y la mayoría tiene acceso a internet, tienen un laboratorio de computación en la institución lo que es significativo para poner en práctica la propuesta planteada, aunque el trabajo con la hoja de cálculo no necesita de una conexión a internet, la misma es importante para mantener el contacto entre los alumnos y el profesor acerca de los ejercicios a realizar.

Finalmente en la dimensión Motivación, en torno a la participación y trabajo con estrategias a través de las hojas de cálculo, se pudo evidenciar en los resultados mostrados en el gráfico 4, en el caso del ítem 4 el cual reflejó que un 92 por ciento consideró que los profesores sí deberían utilizar herramientas tecnológicas, pero un 8 por ciento dice que no. En el ítem 5 un 66 por ciento indicó que sí es factible utilizar una hoja de cálculo para la compresión de la química, pero un 34 por ciento dijo lo contrario.

En el ítem 6 un 58 por ciento afirman que sí están interesados en trabajar con el programa Excel en algunos contenidos de química, pero un 42 por ciento no. Por último en el ítem 7 un 58 por ciento, sí consideran que el uso de una hoja de cálculo facilitaría la comprensión de los contenidos de química, pero un 42 por ciento no.

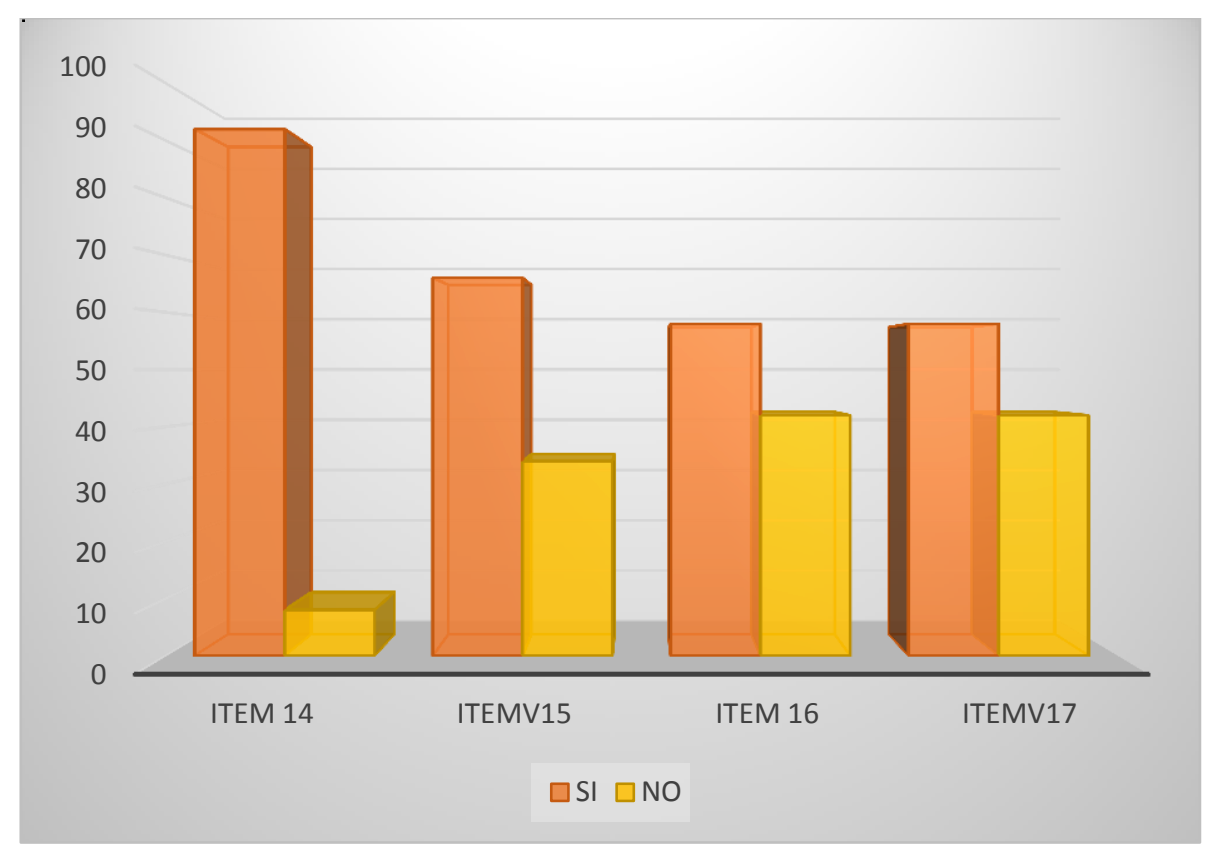

Gráfico 4. Relación porcentual entre los ítem 14, 15, 16, 17. 
Entonces se puede decir, la mayoría de los estudiantes considera que los profesores deberían hacer uso de las herramientas tecnológicas y les gustaría trabajar con ellas, considerando que las mismas facilitarían el contenido que se imparte en las clases de química, con la ayuda de las TIC se constituye a lo que Piaget llamo "andamiaje" un puente entre el conocimiento y el sujeto, lo que indica que se deben generar herramientas que les permita a los alumnos llegar al conocimiento y que puedan asimilar y acomodar su estructura cognitiva; así como, incentivar y renovar la estrategia de aprendizaje para un mayor interés del estudiante hacia la asignatura y lograr así la capacidad de análisis en los problemas prácticos de químicas.

En esta dimensión, los estudiantes destacaron que no utilizan el laboratorio de computación, pero el mismo si está disponible para los docentes que deseen utilizarlo como herramienta para abordar sus clases, aunque la mayoría han utilizado una hoja de cálculo les resulta difícil su manejo, lo que implica que los mismos necesitan capacitación para su uso, aunque en la institución se han utilizados herramientas tecnológicas actualmente no lo hacen, lo que da evidencia que los profesores tienen tendencia impartir sus clases de manera tradicional y se cohíben a hacer uso de las TIC.

El conocimiento de las nuevas tecnologías para la información y comunicación responde al cambio que los estudiantes han tenido radicalmente, se sigue educando en un sistema que ya está obsoleto y se debe ajustar el mismo para dar respuesta a las exigencias de los alumnos de ahora y formarlos en lo que la UNESCO llama "Sociedades del conocimiento" o "de la información" y es aquí donde los docentes deben innovar sus prácticas educativas para darle paso a la era digital para que los estudiantes adquieran habilidades, destrezas y competencias para desenvolverse en la nueva sociedad.

De acuerdo con los resultados, tanto los estudiantes como la institución cuentan con los recursos necesarios para el uso de las herramientas tecnológicas en la asignatura de química y en otras, los mismos tienen la disposición de hacer uso de ellas para mejorar su comprensión en algunos temas y ampliar su conocimiento, sabiendo que la química es muy abstracta, se debe orientar al estudiante a que haga uso de todas las herramientas necesarias para que su aprendizaje sea significativo, los usos de las hojas de cálculo son muchos y se pueden implementar para el aprendizaje de algunos contenidos de química, pero los educadores están más limitados por 
su creatividad que por en conocimiento en programación, Carson (citado en Andrés Raviolo, 2011).

\section{CONCLUSIONES}

i bien es cierto, existe la necesidad de implementar la herramienta (Hoja de cálculo) e innovar el aprendizaje para lograr así un mejor auge en el área de química de la U.E "Hipólito Cisneros" de los estudiantes de cuarto año; tomando en consideración que cuentan con la herramienta principal que es el laboratorio de computación dentro de la Institución. Se encuentra un gran déficit en cuanto al contenido del área de química, generando este un bajo rendimiento en cuanto a resolución de problemas prácticos durante la clase.

Pero es necesario considerar la utilización de las herramientas tecnológicas dentro del área de química, ya que un gran porcentaje de estudiante considera que le interesaría trabajar con el programa Excel siempre y cuando su utilidad sea de forma provechosa y facilite el contenido del área de química y logre consigo resultados satisfactorios en la comprensión del contenido durante la clase.

En atención a la problemática expuesta, la Hoja de cálculo como herramienta didáctica para la comprensión de la cinética química no es de difícil manejo, ni es necesario tener conocimiento de lenguaje de programación, contándose con la herramienta necesaria para su utilización. Visto de esta forma, este trabajo orientó y buscó la forma de desplazar el método tradicionalista rígido arraigado por la innovación y rendimiento durante la soluciones que pudiesen generar inquietudes y desinterés; teniendo como propósito principal motivación hacia el proceso de educativo de los estudiantes; ya que la hoja de cálculo puede ser utilizada por los profesores para la facilitación del contenido durante su desarrollo y así promover el uso de estrategias tecnológicas en la institución permitiendo así la mejora en contenidos.

Vale destacar que también se logró hacer el estudio de la factibilidad la cual permite saber hasta qué punto es posible llevar a cabo un proyecto, se refiere a todos aquellos elementos, técnicas, medios y recursos que se utilizan para la aplicación de los objetivos planteados. De esta forma se demostró la factibilidad económica, técnica y operativa, concluyendo así, la Hoja de cálculo como estrategia didáctica para la compresión de la cinética química, 
dirigido a los estudiantes de 4to año de la U.E. "Hipólito Cisneros" es factible aplicar, ya que existe la necesidad de su aplicación para mejorar las dificultades que presentan los alumnos en el tema de cinética química respondiendo adecuadamente a estas necesidades expuestas con anterioridad.

Como resultado se logró concretar la propuesta la cual buscó promover la inclusión de la tecnología como cambio en el modo de aprendizaje y para la solución de problemas prácticos en la asignatura de química, dirigido a los estudiantes de cuarto año de la U.E. "Hipólito Cisneros. Considerando que la química es una ciencia de origen antiguo que precede de los estudios de alquimia; es una ciencia importante para el mundo, desde el principio de la humanidad el hombre ha necesitado de ella para sobrevivir en la tierra y se encuentra presente en toda materia existente, aunque a los estudiantes les parece un poco difícil por su vocabulario especializado, no es algo que se encuentre lejos de la realidad y que sin saber su naturaleza todos los días se visualiza, por ejemplo; cuando se utiliza una olla de presión para disminuir el tiempo de cocción de los alimentos, cuando se exprime el jugo de limón al pescado para quitar el mal olor, son situaciones en las que se hace uso de la química sin saberlo.

De este mismo modo, el estudio de la cinética química es importante porque permite conocer las diferentes velocidades en las que ocurren las reacciones, algunos procesos como la fotosíntesis suceden a una corta velocidad, pero otros como la conversión del grafito en diamante necesitan millones de años para completarse. La aplicación de este método contribuye en el diseño de fármacos. A través de la Hoja de cálculo como estrategia didáctica para la compresión de la cinética química, se pretendió desarrollar habilidades cognitivas ayudando a una comprensión eficaz de los contenidos que se aspira impartir y de este modo, el docente en aula logre un auge de receptividad continua a través de un enfoqué metodológico diferente que le permita dejar a un lado lo tradicionalista.

Para ello, se estructuró con la ayuda del programa Excel 2013, donde el uso de esta herramienta implicó que los alumnos tenían que construir sus propias tablas programa, los ejercicios son generados por el docente de la asignatura; A continuación se presentan dos ejemplos como referencia: En cuanto a la primera actividad, alusiva a la Tabla 1 denominada: Tabla programa para ilustrar la variación con 
el tiempo del $\mathrm{HCl}$ (ácido clorhídrico); en la imagen colocada, se observa la recopilación de datos que permitieron estudiar el efecto de la concentración del $(\mathrm{HCl})$ dependiendo de la velocidad inicial.

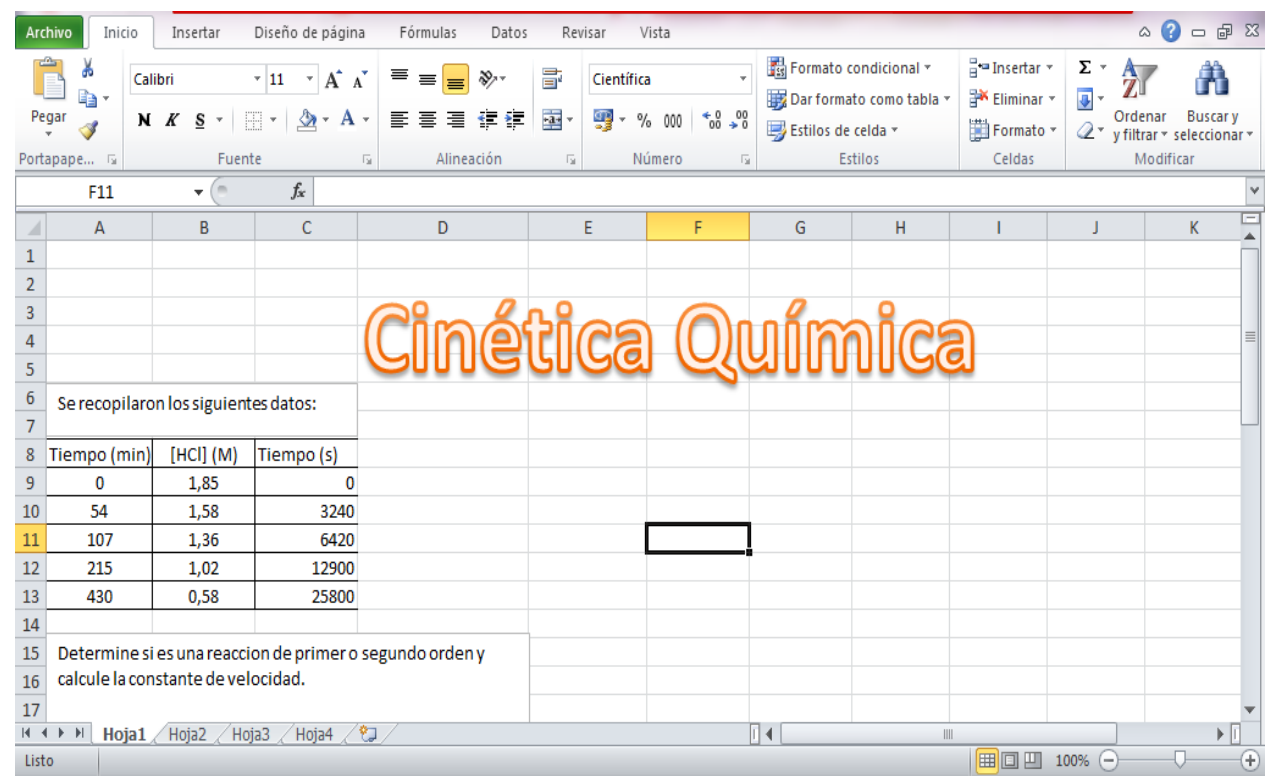

Figura 1. Efecto de la concentración del $(\mathrm{HCl})$ dependiendo de la velocidad inicial

Seguidamente se tiene la siguiente actividad denominada: Secuencia de operaciones programadas; allí se debe determinar el orden de reacción necesaria, la ecuación de velocidad en donde se logró obtener el logaritmo de la concentración, siendo la concentración inicial del reactivo y la concentración pasada en base al tiempo (t). 


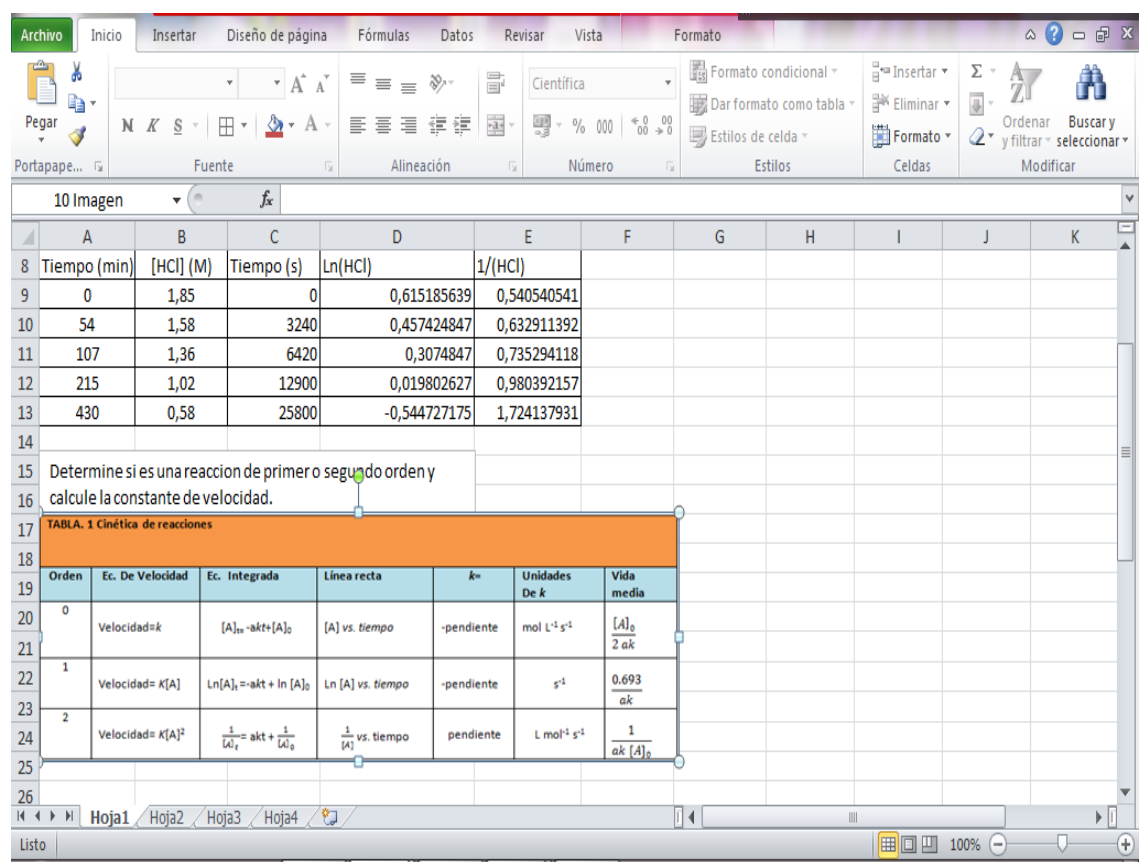

Figura 2. Concentración inicial del reactivo y la concentración pasada en base al tiempo (t)

Finalmente, a través de la Gráfica de segundo orden, se representó el inverso de la concentración y el tiempo expresado en segundo obteniendo la gráfica de segundo orden con un segundo $\mathrm{R}^{2}$, tal como se evidencia a continuación.

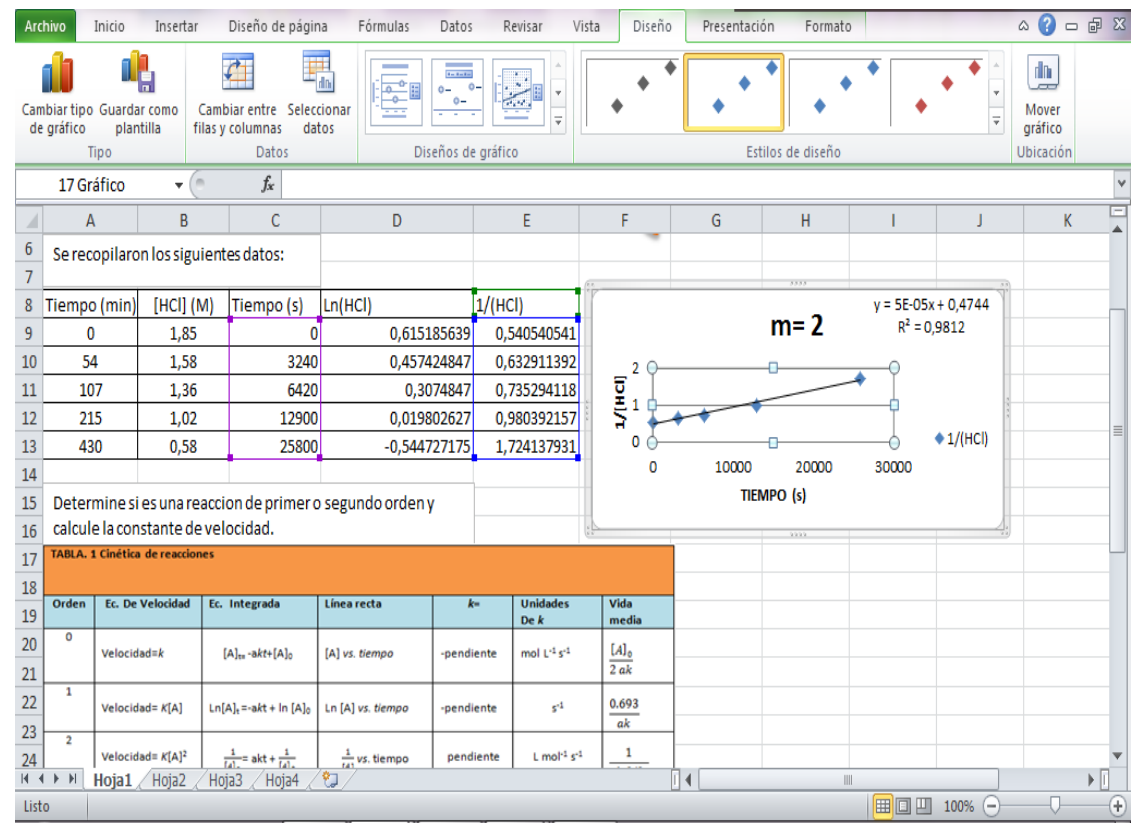

Figura 3. Gráfica de segundo orden con un segundo $\mathrm{R}^{2}$ 


\section{REFERENCIAS}

Arias, F. (2006). El proyecto de investigación: introducción a la metodología científica. (6 edición). Caracas: Episteme

Guffante, T. Guffante, F. y Chávez, P. (2016). Investigación científica: el proyecto de investigación. Recuperado de: https://docplayer.es/ 51150637- El-proyecto-de-investigacion-tania-guffante-naranjofernando-guffante-naranjo-patricio-chavez-hernandez.html

Hurtado, I. y Toro J. (2005). Paradigmas y métodos de la investigación en tiempos de cambio. Recuperado de https://epinvestsite.files. wordpress.com/2017/09/paradigmas-libro.pdf

Palella, S. y Martins, F. (2012). Investigación cuantitativa. (3a ed.) Caracas: FEDUPEL

Raviolo, A. (2011). Enseñanza de la química con la hoja de cálculo. Educación química. Recuperado de: http://dx.doi.org/10.1016/ S0187-893X(18)30157-5

Rojas, E. (2010). Metodología de la investigación. Recuperado de http://metodologia2unefa.blogspot.com/2010/12/antecedentesbases-teoricas-y.html

Hernández, R., Fernández, C y Baptista, M. (2014). Metodología de la investigación cuantitativa. (6 ${ }^{a}$ edición). México: Editorial McGrawHill/ Interamericana de editores S.A De C.V

Tamayo y Tamayo, M. (2003). El proceso de la investigación científica. Recuperado de https://es.scribd.com/doc/12235974/tamayo-ytamayo-mario-el-proceso-de-la-investigacion-cientifica

Unesco. (2013). Enfoques estratégicos sobre las TICS en educación en América Latina y el Caribe. Recuperado de http://www.unesco.org/ new/fileadmin/MULTIMEDIA/FIELD/Santiago/images/ticsesp.pdf 